\title{
Traffic Wave Damping: A Shared Control Approach
}

\author{
Jingjing Jiang ${ }^{1}$, Alessandro Astolfi ${ }^{2}$ and Thomas Parisini ${ }^{3}$
}

\begin{abstract}
Traffic waves, also known as "phantom jams", are caused by the instability of the system describing the dynamics of traffic flow on highways: slight disturbances in the distribution of vehicles on highways are amplified when the density of the traffic is higher than a certain critical value and eventually generate traffic waves and "stop-andgo" phenomena. We propose a solution to the traffic wave damping problem via shared-control on vehicles and show that the effectiveness of the proposed controller does not depend on human drivers' actions. In other terms, with the developed shared-controller the amplitude of the traffic wave is reduced regardless of the action of the drivers. Simulation results on a traffic control benchmark demonstrating the effectiveness of the controller are also provided.
\end{abstract}

\section{INTRODUCTION}

Traffic waves are common phenomena on highways, mainly caused by bottlenecks, such as lane changing [1], [2], merging [3] and accidents. However, vehicles keep accelerating and decelerating even without bottlenecks in crowded sections [4], which has negative impacts on the transportation system: the efficiency is reduced, the total travel time increases andhence people need to spend more time on roads; the carbon emission is increased [5]; and rear-end crashes are more likely to occur [6], [7]. Even worse, the wave usually generates at a congested area with high traffic density and then propagates to non-congested areas [5].

To reduce the above-mentioned side effects, several methods to dampen the wave and to improve the efficiency of the transportation system have been proposed. These have been implemented in freeway controls, such as Ramp Metering (RM) control [8], [9], Variable Speed Limits (VSL) control [10], [11] and the combination of RM and VSL control [12], [13]. Other control methods, such as Model Predictive Control [14] and Iterative Learning Control [15], have also been applied. In addition, recent research works have shown that autonomous vehicles are able to relieve the nuisance caused by traffic waves, to increase the traffic efficiency and

This work has been supported by the European Union's Horizon 2020 research and innovation programme under grant agreement No 739551 (KIOS CoE) and Science and Technology Facilities Council under Newton fund with grant number ST/N006851/1.

${ }^{1} \mathrm{~J}$. Jiang is with the Department of Aeronautical and Automotive Engineering, Loughborough University, LE11 3TU, UK, E-mail: J.Jiang2@lboro.ac.uk

${ }^{2} \mathrm{~A}$. Astolfi is with the Dept. of Electrical and Electronic Engineering, Imperial College London, London, SW7 2AZ, UK, with the KIOS Research and Innovation Center of Excellence, University of Cyprus, and the DICII, University of Roma "Tor Vergata", Via del Politecnico 1, 00133 Rome, Italy, E-mail: a.astolfi@imperial.ac.uk

${ }^{3}$ T. Parisini is with the Dept. of Electrical and Electronic Engineering at Imperial College London, UK, with the KIOS Research and Innovation Center of Excellence, University of Cyprus, and with the Dept. of Engineering and Architecture at University of Trieste, Italy, E-mail: t.parisini@gmail.com to reduce energy consumptions significantly [16]. However, there are two critical issues which are difficult to solve with autonomous vehicles: the first one is that all people become passengers and they cannot enjoy the fun of driving; the other is that to improve the efficiency of the overall transportation system some autonomous vehicles may be slowed down or stopped even if the road in front is clear, which may go against users' satisfaction. To address these issues, this paper proposes a shared-control scheme, rather than an autonomous controller, to dampen traffic waves and improve human drivers' experience.

The main contributions of the paper are stated as follows. The stop-and-go wave simulation that is inspired by the benchmark [17] (see the beginning of Section II) on a circular road is reproduced in the paper. We assume that a supervisory traffic control center, that sends information and recommended actions to the vehicle drivers, is available. Then we propose a shared control scheme [18] to eliminate the traffic wave by making use of information (the recommended speed at the area) sent by the traffic control center, local information detected by on board sensors and the human input on gas and brake. Such a controller does not depend on the human driver and the environment. In other words, the shared controller is such that the amplitude of the traffic is reduced regardless of the action of the drivers and the environments. The theoretical analysis showing the effectiveness of the controller is also given.

This paper is organized as follows. Section II introduces the system dealt with, gives the definition of the human users' satisfaction index and formulates the shared-control problem for damping the stop-and-go wave as well as satisfying human users. The solution to the problem is then given in Section III, in which formal properties of the closedloop system are presented. Simulation results are provided in Section IV to demonstrate the effectiveness of the developed shared controller. Finally, conclusions and future work are discussed in Section V.

\section{NOMENCLATURE}

$T_{s} \quad$ sampling time $[s]$

$n_{c} \quad$ feedback delay samples, i.e. the measurement delay of the feedback controller $T_{F B \text { Delay }}$ is calculated as $T_{F B \text { Delay }}=n_{c} T_{s}$

$n_{d}$ driver's reaction delay samples, i.e. driver's reaction time $T_{\text {rea }}$ is calculated as $T_{r e a}=n_{d} T_{s}$

$a_{h_{i}} \quad$ acceleration of Vehicle $i$ generated by the human driver $\left[\mathrm{m} / \mathrm{s}^{2}\right]$ 


$\begin{array}{ll}a_{c_{i}} & \begin{array}{l}\text { acceleration of Vehicle } i \text { generated by the } \\ \text { feedback controller }\left[\mathrm{m} / \mathrm{s}^{2}\right]\end{array} \\ a_{s_{i}} & \begin{array}{l}\text { acceleration of Vehicle } i \text { generated by the } \\ \text { proposed shared-controller }\left[\mathrm{m} / \mathrm{s}^{2}\right]\end{array} \\ a_{\min } & \begin{array}{l}\text { minimum acceleration of the vehicles }\left[\mathrm{m} / \mathrm{s}^{2}\right] \\ a_{\text {max }}\end{array} \\ d_{\text {min }} & \begin{array}{l}\text { maximum acceleration of the vehicles }\left[\mathrm{m} / \mathrm{s}^{2}\right] \\ \text { minimum distance between two vehicles in order } \\ \text { to avoid collisions }[\mathrm{m}]\end{array} \\ v_{\text {max }} & \begin{array}{l}\text { maximum speed of the vehicles }[\mathrm{m} / \mathrm{s}] \\ \text { recommended speed, given by the higher-level } \\ v^{r}\end{array} \\ v_{i}(k) & \begin{array}{l}\text { traffic control }[\mathrm{m} / \mathrm{s}] \\ \text { speed of Vehicle } i \text { at the time instant } k T_{s}[\mathrm{~m} / \mathrm{s}]\end{array} \\ d_{i, j}(k) & \begin{array}{l}\text { distance between Vehicle } i \text { and Vehicle } j \text { at the } \\ \text { time instant } k T_{s}[\mathrm{~m}]\end{array} \\ f_{i}(k) \quad \begin{array}{l}\text { value of the sharing function for Vehicle } i \text { at the } \\ \text { time instant } k T_{s}\end{array} \\ \mathcal{S}_{i}(k) \quad \begin{array}{l}\text { satisfaction index for the driver of Vehicle } i \text { at } \\ \text { the time instant } k T_{s}\end{array} \\ x_{i}(k) \quad \begin{array}{l}\text { position of Vehicle } i \text { at the time instant } k T_{s}[\mathrm{~m}]\end{array}\end{array}$

\section{SYSTEM DESCRIPTION AND PROBLEM FORMULATION}

This section describes the setup of the traffic control scenario, gives the car-following model representing the human drivers and formulates the shared-control problem for the traffic wave damping.

\section{A. System Description}

The traffic wave phenomenon has been studied in [5], [19] and it has been recently reproduced experimentally on a circular road in [17]. This paper uses the same setup as that given in [17]: 21 vehicles, denoted as Vehicle 1, Vehicle $2, \ldots$, Vehicle 21 (also denoted as Vehicle 0), are driven on a single-lane circular road with radius of 41.4 meters. The distance between Vehicle 1 and its preceding vehicle, Vehicle 21, is denoted as $d_{0,1}$. Note that we use the same benchmark for simplicity and comparison purposes. However, there is no loss of generality because the approach is valid for different configurations.

Assumption 1: The vehicles are unable to move backward and the maximum forward speed for all vehicles, denoted as $v_{\max }$, is the same. In addition, the minimum acceleration for all vehicles, denoted as $a_{m i n}$, is negative and constant, while the maximum acceleration for all vehicles, denoted as $a_{\max }$, is positive and constant.

Remark 1: In the setup described in this subsection the lane is supposed homogeneous. In addition, there is no leading vehicle and each car follows its preceding one.

\section{B. Car-Following Model}

In the rest of the paper we use the following nonlinear dynamics to describe the car-following model given in [20]:

$$
\begin{aligned}
& x_{i}(k+1)=x_{i}(k)+T_{s} v_{i}(k), \\
& v_{i}(k+1)=v_{i}(k)+T_{s} a_{h_{i}}(k),
\end{aligned}
$$

where the definitions of $x_{i}(k), v_{i}(k)$ and $T_{s}$ are given in the Nomenclature and $a_{h_{i}}(k)$ is calculated as

$$
a_{h_{i}}(k)=\min \left\{\begin{array}{c}
\max \left[a_{i, c a l}(k), a_{\min },-\frac{v_{i}(k)}{T_{s}}\right], \\
m_{i}(k), a_{\max }, \frac{v_{\max }-v_{i}(k)}{T_{s}}
\end{array}\right\},
$$

with

$$
\begin{aligned}
a_{i, c a l}(k)= & C_{i, 2}\left[d_{i-1, i}\left(k-n_{d}\right)-d_{\min }-\beta v_{i}\left(k-n_{d}\right)\right] \\
& +C_{i, 1}\left[v_{i-1}\left(k-n_{d}\right)-v_{i}\left(k-n_{d}\right)\right],
\end{aligned}
$$

and

$$
m_{i}(k)=\frac{d_{i-1, i}(k)}{T_{s}^{2}}+\frac{v_{i-1}(k)-2 v_{i}(k)}{T_{s}}-\frac{d_{\min }}{T_{s}^{2}} .
$$

Note that $d_{i-1, i}(k)$ represents the distance between Vehicle $i-1$ and Vehicle $i$ at the time instant $k T_{s}$, while the definitions of $n_{d}$ and $d_{\min }$ are given in the Nomenclature. In addition, $C_{i, 1}$ and $C_{i, 2}$ are two positive constants describing the driver's behaviour when following the preceding vehicle. Generally speaking, both of these vary according to the driver, the weather, the road surface, etc. Typical values are given as: $C_{i, 1}=0.5, C_{, 2}=0.125, d_{\min }=5, \beta=2, n_{d}=$ 15 and $T_{s}=0.1$ (see [17], [21]).

We assume that the following assumptions hold for the rest of the paper.

Assumption 2: $a_{h_{i}}(k)=0$, for all $k \in\left\{0,1, \ldots, n_{d}-1\right\}$ and all $i \in\{1,2, \ldots, 21\}$.

Assumption 2 indicates that if the vehicles are fully controlled by human drivers, then the speed of all the vehicles does not change during the initial few samples because of the drivers' reaction delay. Therefore $v_{i}(0)=v_{i}(1)=\cdots=$ $v_{i}\left(n_{d}-1\right), x_{i}(k)=x_{i}(0)+k T_{s} v_{i}(0)$ and $d_{i-1, i}(k)=$ $d_{i-1, i}(0)+k T_{s}\left(v_{i-1}(0)-v_{i}(0)\right)$, for all $k \in\left\{0,1, \ldots, n_{d}-\right.$ $1\}$ and all $i \in\{1,2, \ldots, 21\}$.

Assumption 3: The initial position, $x_{i}(0)$, and velocity, $v_{i}(0)$, of Vehicle $i$ are chosen such that all safety constraints and mechanical constraints are satisfied for a certain period, i.e. $0 \leq v_{i}(0) \leq v_{\max }$ and $d_{\min } \leq d_{i-1, i}(k)+T_{s} v_{i}(0)$, for all $i \in\{1,2, \ldots, 21\}$ and $k \in\left\{0,1, \ldots, n_{d}-1\right\}$.

Since human drivers have reaction delays, Assumption 3 is essential to guarantee that all safety and mechanical constraints are satisfied before the human driver is able to take any action.

The properties of the system when all vehicles are controlled completely by human drivers are given in Proposition 1, from which we can conclude that the car-following model we used is able to satisfy all the mechanical constraints and safety constraints.

Proposition 1: Consider a vehicle set $\{$ Vehicle 1, Vehicle $2, \ldots$, Vehicle 21$\}$ describing a platoon of 21 homogeneous vehicles. The relationship between adjacent vehicles is described by the car-following model given in (1)-(2). Let 

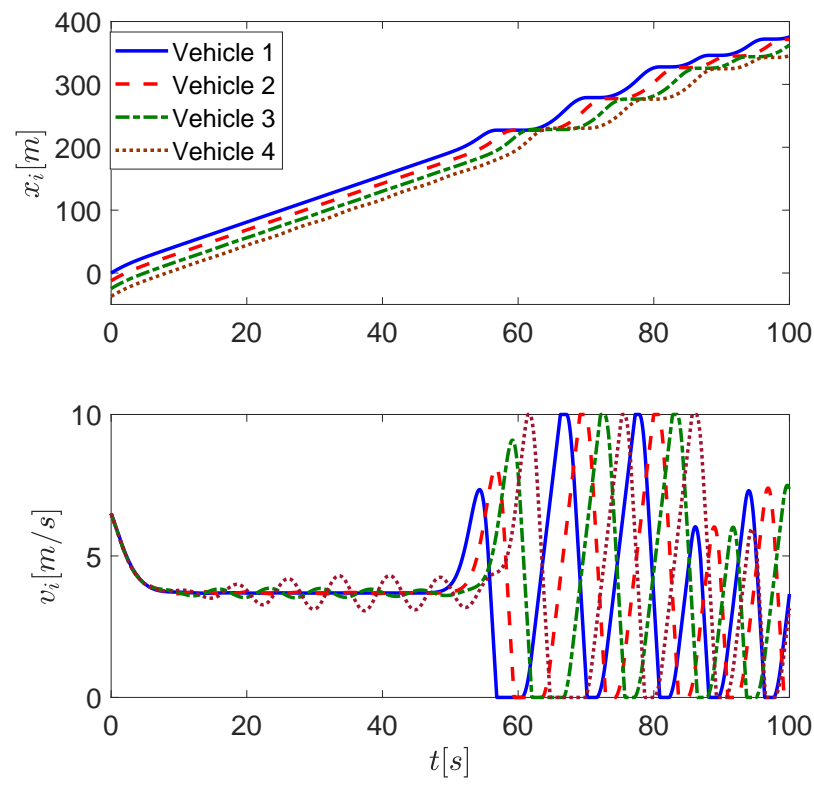

Fig. 1: Time histories of the travelled distances and speeds of Vehicles 1, 2, 3 and 4.

Assumptions 2 and 3 hold. Then the mechanical constraints and safety constraints

C1) $v_{i}(k) \in\left[0, v_{\max }\right]$, for all $i \in\{1,2, \ldots, 21\}$ and $k \in\{1,2, \ldots\}$;

C2) $a_{h_{i}}(k) \in\left[a_{\min }, a_{\max }\right]$, for all $i \in\{1,2, \ldots, 21\}$ and $k \in\{1,2, \ldots\}$;

C3) $x_{i-1}(k)-x_{i}(k+1) \geq d_{\text {min }}$, for all $i \in\{1,2, \ldots, 21\}$ and $k \in\{1,2, \ldots\}$;

are satisfied.

Due to the lack of space, the proofs of the proposition, lemma and theorem given in the paper are omitted.

Remark 2: We have chosen the car-following model given by (1) not only because it satisfies all the mechanical and safety constraints, but also because it is able to generate the same traffic wave observed in experiments as detailed in [17].

Assume that all the vehicles are controlled by human drivers and that, initially, the vehicles are evenly distributed along the path, i.e. the distance between two adjacent vehicles is $12.4 \mathrm{~m}$, and they have the same speed of $6.5 \mathrm{~m} / \mathrm{s}$. By running the simulation we observe that the stop-and-go wave is generated within $100 s$, as illustrated in Fig. 1: Vehicle 1 is almost stationary during the time periods $\left[\begin{array}{llll}56 & s, 63 & s\end{array}\right]$, $\left[\begin{array}{lll}69 & s, 74 & s\end{array}\right],\left[\begin{array}{llll}80 & s, 84 & s\end{array}\right]$ and $\left[\begin{array}{llll}88 & s, 91 & s\end{array}\right]$ as the curve representing the time histories of the travelled distance is flat and the speed drops to zero during these periods. In addition, even though we have only plotted variables associated to four vehicles, all the other vehicles have similar behaviour.

\section{Problem Statement}

Shared-control refers to a control scheme which combines the human driver's input together with an input generated by a feedback controller. Before formulating the sharedcontrol problem, we need to define how the shared-control is able to combine the human driver's input and the feedback control input. In addition, we need to define the satisfaction index describing how satisfied the human driver is with the proposed shared controller. These are given as follows.

Definition 1: We use the name $h$-control, c-control and $s$ control, denoted as $a_{h_{i}}, a_{c_{i}}$ and $a_{s_{i}}$, to describe the human action, the feedback control action and the shared-control action for Vehicle $i$, respectively. In addition, we use the name sharing function, denoted as $f_{i}$, to quantify how the control authority is shared between the c-control and the hcontrol, i.e.

$$
a_{s_{i}}=\left(1-f_{i}\right) a_{c_{i}}+f_{i} a_{h_{i}} .
$$

Definition 2: Consider the driver of Vehicle $i$ and his/her estimation of the speed of the preceding vehicle at the time instant $k T_{s}$, i.e. $v_{i-1}\left(k-n_{d}\right)$. The satisfaction index $\mathcal{S}_{i}(k)$ for the driver of Vehicle $i$ at the time instant $k T_{s}$, is defined as

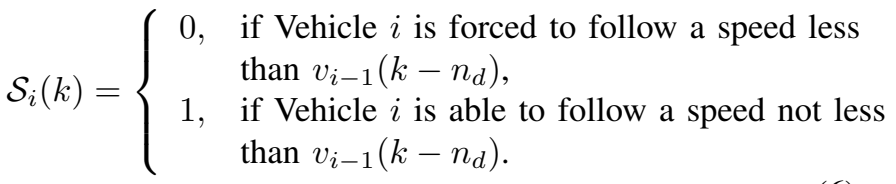

Note that $\mathcal{S}_{i}(k)=0\left(\mathcal{S}_{i}(k)=1\right)$ indicates that the driver of Vehicle $i$ is disappointed (satisfied) with the actual situation at the time instant $k T_{s}$. The shared-control problem for traffic wave damping can then be formulated as follows.

Consider a fleet of 21 homogeneous vehicles controlled by the shared controller, with the dynamics of the Vehicle $i$ described by the equations

$$
\begin{aligned}
& x_{i}(k+1)=x_{i}(k)+T_{s} v_{i}(k), \\
& v_{i}(k+1)=v_{i}(k)+T_{s} a_{s_{i}}(k),
\end{aligned}
$$

where $a_{s}$ is defined in (5). Assume that the human drivers' behaviour is modeled as (1)-(2) and Assumptions 1 to 3 hold. The objective is to find (if possible)

- a c-control $a_{c_{i}}$

- a sharing function $f_{i}$

for each Vehicle $i$, such that the overall closed-loop system with 21 vehicles (the dynamics of Vehicle $i$ is described by equations (2)-(5)-(7) for all $i \in\{1,2, \ldots, 21\}$ ) has the following properties.

P1) The stop-and-go wave is damped, i.e. there exists a signal $v_{\min }(k)$, the value of which is always positive, such that $v_{i}(k) \in\left[v_{\min }(k), v_{\max }\right]$, for all $i \in\{1,2, \ldots, 21\}$ and $k \in\{1,2, \ldots\}$. 
P2) The saturation constraints on accelerations are always satisfied, i.e. $a_{s_{i}}(k) \in\left[a_{\min }, a_{\max }\right]$, for all $i \in\{1,2, \ldots, 21\}$ and $k \in\{1,2, \ldots\}$.

P3) The distance between two adjacent vehicles is always greater than a safety-guarantee distance, i.e. $x_{i-1}(k)-x_{i}(k+1) \geq d_{\text {min }}$, for all $i \in\{1,2, \ldots, 21\}$ and $k \in\{1,2, \ldots\}$.

P4) The driver is always "happy", i.e. $\mathcal{S}_{i}(k)=1$, for all $i \in\{1,2, \ldots, 21\}$ and $k \in\{1,2, \ldots\}$.

\section{SHARED-CONTROL DESIGN TO DAMPEN TRAFFIC WAVES}

This section provides a solution to the shared control problem stated in Section II-C, followed by the analysis of the properties of the resulting closed-loop system.

The shared control contains two parts: the c-control and the sharing law. Furthermore, the design of the shared control is based on information from the environment (including the speed of the vehicle, the distance and the relative speed between the vehicle and the preceding one), the recommended speed from a higher level controller and the human driver's input.

\section{A. Design of the c-control}

This subsection provides the design of the c-control as the first step in the shared control design. The c-control is similar to the car-following dynamics representing the human behaviour. Therefore, the dynamics of the Vehicle $i$ controlled solely by the c-control can be described by the equations

$$
\begin{aligned}
& x_{i}(k+1)=x_{i}(k)+T_{s} v_{i}(k), \\
& v_{i}(k+1)=v_{i}(k)+T_{s} a_{c_{i}}(k),
\end{aligned}
$$

where

$$
a_{c_{i}}(k)=\min \left\{\begin{array}{c}
\max \left[a_{c c_{i}}(k), a_{\min },-\frac{v_{i}(k)}{T_{s}}\right], \\
m_{i}(k), a_{\max }, \frac{v_{\max }-v_{i}(k)}{T_{s}}
\end{array}\right\},
$$

with

$$
\begin{aligned}
a_{c c_{i}}(k)= & C_{c 2}\left[\begin{array}{c}
x_{i-1}\left(k-n_{c}\right)-x_{i}\left(k-n_{c}\right) \\
-D_{i}\left(k-n_{c}\right)
\end{array}\right] \\
& +C_{c 1}\left[v^{r}-v_{i}\left(k-n_{c}\right)\right], \\
D_{i}\left(k-n_{c}\right)= & d_{m i n}+\beta v_{i}\left(k-n_{c}\right),
\end{aligned}
$$

and $m_{i}(k)$ given in (4).

Significant work has been done to calculate the reference speed, also regarded as the speed limit at highways, to reduce congestions, see e.g. [11], [22], [23], [24]. Note that the recommended speed $v^{r}$ is usually related to the density of the traffic.

Assumption 4: $a_{c_{i}}(k)=0$, for all $k \in\left\{0,1,2, \ldots, n_{c}-1\right\}$ and all $i \in\{1,2, \ldots, 21\}$. In addition, for any given $n_{c}, C_{c 1}$ and $C_{c 2}$, the initial location and speed of Vehicle $i$ is chosen such that the constraints

- $\frac{C_{c 1}}{C_{c 1}+C_{c 2} \beta} v^{r}+a_{\min } T_{s}\left(n_{c}+1\right) \leq v_{i}(0) \leq v_{\max }$ for all $i \in\{1,2, \ldots, 21\}$;

- $d_{\text {min }} \leq x_{i-1}(0)-x_{i}(0)+k T_{s}\left[v_{i-1}(0)-v_{i}(0)\right]-$ $T_{s} v_{i}(0)$, for all $i \in\{1,2, \ldots, 21\}$ and $k \in$ $\left\{0,1,2, \ldots, n_{c}-1\right\}$;

hold, where $v^{r}$ is a constant value.

Note that Assumption 4 is given to guarantee that P1) and P3) in Section II-C hold before the feedback controller is able to take any action. In addition, assuming $v^{r}$ to be constant is not very restrictive, since the rate of change of $v^{r}$ given by the traffic control center is typically very slow compared to the wave dynamics.

Lemma 1: Consider the system (8) in closed-loop with (9). Suppose Assumptions 1 and 4 hold. Then the closed-loop system has the following properties:

- $v_{i}(k) \in\left[\frac{C_{c 1}}{C_{c 1}+C_{c 2} \beta} v^{r}+a_{\min } T_{s}\left(n_{c}+1\right), v_{\max }\right]$, for all $i \in\{1,2, \ldots, 21\}$ and $k \in\{1,2, \ldots\}$;

- $a_{s_{i}}(k) \in\left[a_{\min }, a_{\max }\right]$, for all $i \in\{1,2, \ldots, 21\}$ and $k \in\{1,2, \ldots\}$;

- $x_{i-1}(k)-x_{i}(k+1) \geq d_{m i n}$, for all $i \in\{1,2, \ldots, 21\}$ and $k \in\{1,2, \ldots\}$.

Remark 3: Lemma 1 shows that P1) to P3) in Section II$\mathrm{C}$ hold if all vehicles are fully controlled by the feedback controller (9).

For typical applications, the communication delay $n_{c}$ is much smaller compared to the driver's reaction time $n_{d}$. Typical values chosen in the design of the $c$-control are given as: $C_{c 1}=0.5, C_{c 2}=0.125$ and $n_{c}=2$. In addition, the parameter values for vehicles describing the constraints of the mechanical system are: $a_{\max }=2, a_{\min }=-4$ and $v_{\max }=10$ (see [17], [25]).

\section{B. Shared-Control Law and Properties of the Closed-Loop System}

This subsection provides a scheme to combine the feedback controller $a_{c_{i}}$ and the human input $a_{h_{i}}$ aiming at eliminating the stop-and-go wave as well as to satisfy the human driver.

The sharing function of Vehicle $i$ at the time instant $k T_{s}$, namely the function $f_{i}(k)$, is defined as

$$
f_{i}(k)= \begin{cases}1, & \sigma_{1} \leq v_{i-1}\left(k-n_{d}\right)-v^{r}\left(k-n_{c}\right), \\ l_{i}(k), & \sigma_{2}<v_{i-1}\left(k-n_{d}\right)-v^{r}\left(k-T_{c}\right)<\sigma_{1}, \\ 0, & v_{i-1}\left(k-n_{d}\right)-v^{r}\left(k-n_{c}\right) \leq \sigma_{2},\end{cases}
$$


where $l_{i}(0)=1$ and

$$
l_{i}(k)= \begin{cases}1, & \text { if } f_{i}(k-1)=1 \\ 0, & \text { if } f_{i}(k-1)=0\end{cases}
$$

for all $k \in\{1,2, \ldots\}$. In addition, $\sigma_{1}$ and $\sigma_{2}$ are two userselected constants with $\sigma_{2}<\sigma_{1}$.

Clearly the sharing function looks like a hysteresis switch, which is able to reduce the oscillations [18]. In order to make the satisfaction index defined in (6) equal to 1 , we choose $\sigma_{1}=0$ and $\sigma_{2}$ to be a negative constant selected by the driver. Note that the smaller the value of $\sigma_{2}$ is chosen the less aggressive the driver is, because this allows the feedback controller to work for a broader range of situations. Otherwise, if $\sigma_{2}$ is a large negative constant, the driver tends to follow the information sent by the higher level control and allows the feedback controller to gain control authority of the vehicle when the recommended speed $v^{r}\left(k-n_{c}\right)$ is slightly above the estimated speed of the preceding vehicle, i.e. $v_{i-1}\left(k-n_{d}\right)$.

Finally, the overall shared-control law for Vehicle $i$ at the time instant $k T_{s}, a_{s_{i}}(k)$, is given by (5) where $f_{i}(k), a_{c_{i}}(k)$ and $a_{h_{i}}(k)$ are calculated by (10), (9) and (2), respectively.

The properties of the closed-loop system (2)-(5)-(7)-(9)-(10) are summarized in the following important result.

Theorem 1: Consider the closed-loop system (2)-(7) controlled by (5)-(9)-(10). Suppose Assumptions 1 to 4 hold and the constant recommended speed $v^{r}(k)$ is such that $\frac{C_{c 1}}{C_{c 1}+C_{c 2} \beta} v^{r}(k)+a_{\min } T_{s}\left(\max \left(n_{c}, n_{d}\right)+1\right)>0$, for all $k \in\{1,2, \ldots\}$. Let $a_{h_{i}}$ be an unknown h-control ${ }^{1}$ for all $i \in\{1,2, \ldots, 21\}$ such that Proposition 1 holds. Then for any negative user-selected $\sigma_{2}$ and zero $\sigma_{1}$, the closed-loop system has the following properties.

P1) No vehicle stops, i.e. there exists a positive signal $v_{\min }(k)$ such that $v_{i}(k) \in\left[v_{\min }(k), v_{\max }\right]$, for all $i \in\{1,2, \ldots, 21\}$ and $k \in\{1,2, \ldots\}$.

P2) Mechanical constraints on accelerations are satisfied, i.e. $a_{s_{i}}(k) \in\left[a_{\min }, a_{\max }\right]$, for all $i \in\{1,2, \ldots, 21\}$ and $k \in\{1,2, \ldots\}$.

P3) There is no collision, i.e. $x_{i-1}(k)-x_{i}(k+1) \geq d_{\text {min }}$, for all $i \in\{1,2, \ldots, 21\}$ and $k \in\{1,2, \ldots\}$.

P4) The human driver is always satisfied, i.e. $\mathcal{S}_{i}(k)=1$, for all $i \in\{1,2, \ldots, 21\}$ and $k \in\{1,2, \ldots\}$.

\section{SIMULATION RESULTS}

This section provides simulation results for the system described in Section II with and without the proposed shared controller. The comparison shows the effectiveness of the shared controller.

As described in Section II, the initial distance between two adjacent vehicles is set to 12.4 meters, which equals to the

\footnotetext{
${ }^{1}$ We assume that the parameter values of the car-following model, i.e. $C_{i, 1}$ and $C_{i, 2}$, are unknown constants in certain ranges, i.e. $C_{i, 1} \in$ $\left[\underline{C}_{1}, \bar{C}_{1}\right]$ and $C_{i, 2} \in\left[\underline{C}_{2}, \bar{C}_{2}\right]$.
}
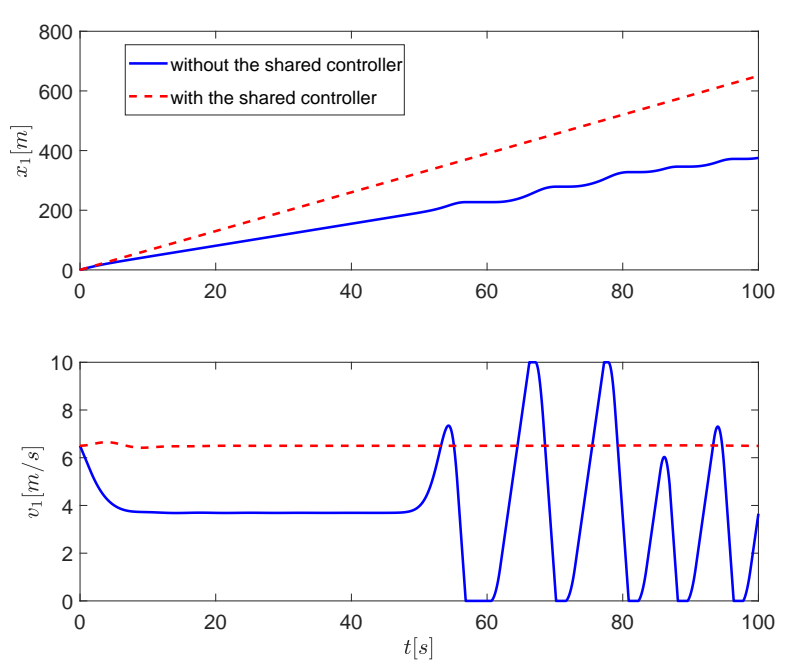

Fig. 2: Comparison between the travelled distance and the velocity of Vehicle 1 with (red, dashed line) and without (blue, solid line) shared-control.

equilibrium distance, while the initial speed and acceleration for all vehicles are set to $6.5 \mathrm{~m} / \mathrm{s}$ and $0 \mathrm{~m} / \mathrm{s}^{2}$, respectively. In addition, the recommended speed $v^{r}$ is set to $6.5 \mathrm{~m} / \mathrm{s}$.

In the simulation, we have chosen $\sigma_{2}=-2 \mathrm{~m} / \mathrm{s}$ and the simulation results are given in Figs. 2 and 3. Fig. 2 shows that the shared controller is able to eliminate the stop-andgo wave as the speed of Vehicle 1 is always positive and there is no flat segment in the curve displaying the time history of $x_{1}$. It also shows that with the help of the shared controller the total travelled distance for Vehicle 1 within $100 \mathrm{~s}$ is about $650 \mathrm{~m}$. Compared with that without the shared controller, i.e. $370 \mathrm{~m}$, the average speed with the proposed control scheme has increased by $75 \%$. Fig. 3 illustrates the distance between several selected adjacent vehicles: the distance varies between $5 \mathrm{~m}$ and $30 \mathrm{~m}$ without the shared controller, while it varies between $11.8 \mathrm{~m}$ and $13.2 \mathrm{~m}$, initially, and then drops to $12.4 \mathrm{~m}$, i.e. the equilibrium distance, later on if the shared controller is used. Therefore, the proposed shared controller is able to dampen the traffic wave.

\section{CONCLUSIONS}

This paper has shown how to address the traffic wave damping problem generated by 21 vehicles being driven on a circular road according to a well-known benchmark. The solution is based on a shared control scheme which combines the action of a feedback controller together with the action of the human driver. Such controller aims to reduce the wave, to improve the efficiency and to satisfy the human driver. Formal properties of the closed-loop system are established. In addition, the theoretical analysis shows that the effectiveness of the controller does not depend on the selection of the parameter values in the car-following model describing the human driver's behaviour. This indicates that 
(a) with the shared controller
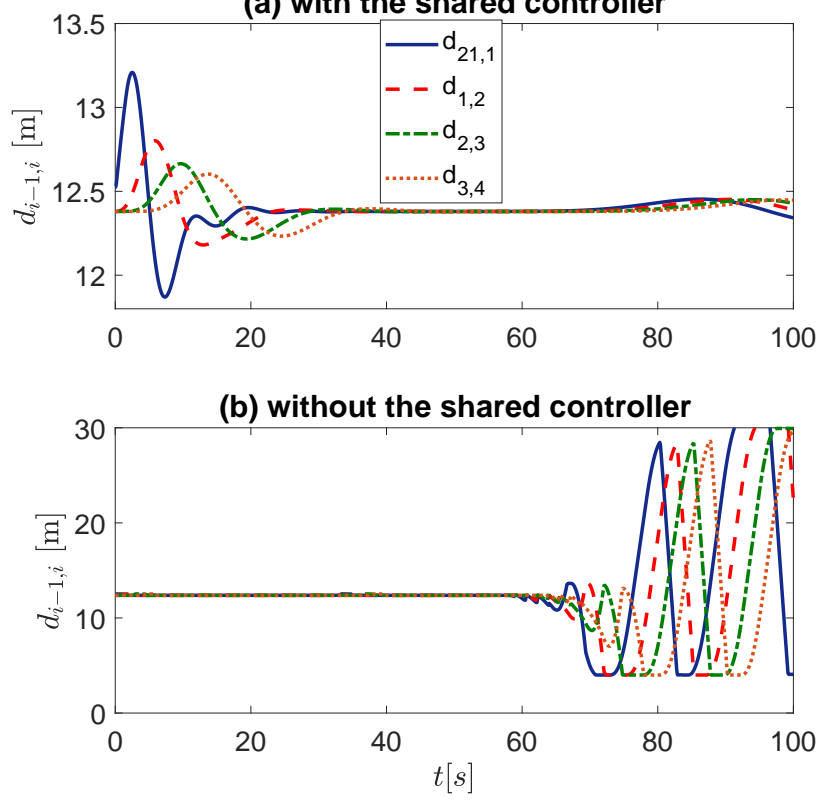

Fig. 3: Distances between adjacent vehicles without (a) and with (b) shared-control. $v^{r}$ is set to $8.4 \mathrm{~m} / \mathrm{s}$.

the shared controller works for different people driving habits and different kinds of vehicle. Moreover, the simulation results in Section IV demonstrate the effectiveness of the shared controller. In the future, we will study how the shared controller can be implemented on a circular path with entrances, exits and multiple lanes. Such simulation scenario setup allows lane-changing maneuvers and allows vehicles to travel in and out of the system.

\section{REFERENCES}

[1] S. Ahn and M. Cassidy, "Freeway traffic oscillations and vehicle lanechange maneuvers," in Proc. of Transportation and Traffic Theory, 2007, pp. 691-710.

[2] A. Ferrara, S. Sacone, and S. Siri, Freeway Traffic Modelling and Control. New York, USA: Springer International Publishing, 2018.

[3] D. Ni and J. Leonard, "A simplified kinematic wave model at a merge bottleneck," Applied Mathematical Modelling, vol. 29, no. 11, pp. 1054-1072, 2005.

[4] G. Orosz, R. Wilson, R. Szalai, and G. Stépán, “Traffic jams without bottlenecks: experimental evidence for the physical mechanism of the formation of a jam," New Journal of Physics, vol. 10, p. 033001, 2008.

[5] J. Laval and L. Leclercq, "A mechanism to describe the formation and propagation of stop-and-go waves in congested freewaytraffic," Philosophical Trans. on the Royal Society A, vol. 368, pp. 4519-4541, 2010.

[6] T. Golob, W. Recker, and Y. Pavlis, "Probabilistic models of freeway safety performance using traffic flow data as predictors," Safety Science, vol. 46, no. 9, pp. 1306-1333, 2008.

[7] P. Marchesini and W. Weijermars, The Relationship Between Road Safety and Congestion on Motorways. Leidschendam, The Netherlands: SWOV Institute for Road Safety Research, 2010.

[8] I. Papamichail, A. Kotsialos, I. Margonis, and M. Papageorgiou, "Coordinated ramp metering for freeway networks: A model-predictive hierarchical control approach," Transportation Research Part C: Emerging Technologies, vol. 18, no. 3, pp. 311-331, 2010.

[9] G. Gomes and R. Horowitz, "Optimal freeway ramp metering using the asymmetric cell transmission model," Transportation Research Part C: Emerging Technologies, vol. 14, no. 4, pp. 244-262, 2006.
[10] A. Hegyi, S. Hoogendoorn, M. Schreuder, H. Stoelhorst, and F. Viti, "SPECIALIST: A dynamic speed limit control algorithm based on shock wave theory," in Proc. of IEEE Conference on Intelligent Transportation Systems, Beijing, China, 2008, pp. 827-832.

[11] R. Carlson, I. Papamichail, and M. Papageorgiou, "Local feedbackbased mainstream traffic flow control on motorways using variable speed limits," IEEE Trans. on Intelligent Transportation Systems, vol. 12, no. 4, pp. 1261-1276, 2011.

[12] _ - "Integrated feedback ramp metering and mainstream traffic flow control on motorways using variable speed limits," Transportation Research Part C: Emerging Technologies, vol. 46, pp. 209-221, 2014.

[13] R. Carlson, I. Papamichail, M. Papageorgiou, and A. Messmer, "Optimal motorway traffic flow control involving variable speed limits and ramp metering," Transportation Science, vol. 44, no. 2, pp. 238-253, 2010.

[14] A. Hegyi, B. Schutter, and H. Hellendoorn, "Model predictive control for optimal coordination of ramp metering and variable speed limits," Transportation Research Part C: Emerging Technologies, vol. 13, no. 3, pp. 185-209, 2005.

[15] Z. Hou, J. Xu, and H. Zhong, "Freeway traffic control using iterative learning control-based ramp metering and speed signaling," IEEE Trans. on Vehicular Technology, vol. 52, no. 2, pp. 466-477, 2007.

[16] S. Gong, A. Zhou, J. Wang, T. Li, and S. Peeta, "Cooperative adaptive cruise control for a platoon of connected and autonomous vehicles considering dynamic information flow topology," in Proc. of IEEE Conference on Intelligent Transportation Systems, 2018, pp. 1-6.

[17] R. Stern, S. Cui, M. Monache, R. Bhadani, M. Bunting, M. Churchill, N. Hamilton, R. Haulcy, H. Pohlmann, F. Wu, B. Piccoli, B. Seibold, J. Sprinkle, and D. Work, "Dissipation of stop-and-go waves via control of autonomous vehicles: Field experiments," Transportation Research Part C: Emerging Technologies, vol. 89, pp. 205-221, 2018.

[18] J. Jiang, P. Franco, and A. Astolfi, "Shared control for the kinematic and dynamic models of a mobile robot," IEEE Trans. on Control Systems Technology, vol. 24, no. 6, pp. 2112-2124, 2016.

[19] Y. Sugiyama, M. Fukui, M. Kikuchi, K. Hasebe, A. Nakayama, K. Nishinari, S. Tadaki, and S. Yukawa, "Traffic jams without bottlenecks: experimental evidence for the physical mechanism of the formation of a jam," New Journal of Physics, vol. 10, no. 3, p. 033001, 2008. [Online]. Available: http://stacks.iop.org/1367$2630 / 10 / \mathrm{i}=3 / \mathrm{a}=033001$

[20] W. Helly, "Simulation of bottlenecks in single lane traffic flow," in Proc. of Symposium on Theory of Traffic Flow, 1959, pp. 207-238.

[21] M. Brackstone and M. McDonald, "Car-following: A historical review," Transportation Research Part F: Traffic Psychology and Behaviour, vol. 2, no. 4, pp. 181-196, 1999.

[22] Z. Li, P. Liu, C. Xu, H. Duan, and W. Wang, "Reinforcement learningbased variable speed limit control strategy to reduce traffic congestion at freeway recurrent bottlenecks," IEEE Transactions on Intelligent Transportation Systems, vol. 18, no. 11, pp. 3204-3207, 2017.

[23] K. Kang and G. Chang, "A robust model for optimal time-of-day speed control at highway work zones," IEEE Transactions on Intelligent Transportation Systems, vol. 7, no. 1, pp. 115 - 123, 2006.

[24] X. Yang, Y. Lin, Y. Lu, and N. Zou, "Optimal variable speed limit control for real-time freeway congestions," in Proc. of International Conference of Transportation Professionals, 2013, pp. 2362-2372.

[25] A. . Bartlett, J.S. (2018, "Best and worst car acceleration," in Retrieved from https://www.consumerreports.org/cars-best-and-worstcar-acceleration. 